\title{
Wireless Personal Safety Bracelet
}

\author{
Apurva Deshmukh \\ E \& TC Dept. SITRC \\ Nashik Maharastra, India
}

\author{
Amit Kumar Mishra \\ E \& TC Dept. SITRC \\ Nashik Maharastra, India \\ Kundan Saraf \\ E \& TC Dept. SITRC \\ Nashik Maharastra, India
}

\author{
Vijay Patil \\ E \& TC Dept. SITRC \\ Nashik Maharastra, India
}

\begin{abstract}
An accident could stop a business in seconds. So prevent it from happening and always turn on safety awareness button before you start.

This bracelet bundles triple-tech Real-Time Location System (RTLS) technologies to deliver precise real time positioning data so that a host RTLS safety application or an integrated physical security platform can track the where about of the individual anywhere within the facility. The bracelet features a large duress call button designed especially for healthcare patients and senior citizens. Pressing the button causes the tag to transmit positioning data that identifies the person requiring assistance and the precise building location of the involving medical or safety incident for speedier and more effective response efforts.
\end{abstract}

\section{Keywords}

Time Location System; Super Heterodyne Receiver; Visual Basic.NET; MsSQL; XC2S50-5TQ144; Pentium4 processor; unique ID.

\section{INTRODUCTION}

We are creating a safe environment for women and children everywhere. The use of this bracelet will reduce the number of women assaulted and children abducted. Our goal is to make affordable for every women and/ or parent regardless of income .protection from assailants, bullies, attempted date rape, child predators, and many other dangerous situations.

We all find ourselves in situations that at times seem hopeless. And, we all have the choice to do nothing or to take action your action could save lives so don't be bystander when it comes to Safety .If you are not safety conscious you could end up unconscious so think about it. we will judge my success in life by how many people feel safe enough to cry in my arms.

By using this bracelets, women will be secured and many lives will be prevented from abusing. As, safety will di erentiate success and failure.

Not only women but this product will be useful for senior citizens, in case of health security, small children for knowing whether they are properly dropped in school, tuition or not, by pressing a button or message can come to know just sitting in home ,in jails if any theft try to break that locked bracelet the buzzer will alert all main staff.

\section{REVIEW OF LITERATURE}

There are various apps developed for women. Designed for college students, Circle of 6 is also useful for high school students or any female of any age who wants an easy-to-use system to alert friends when she's in a threatening situation. As fully functional as those apps which require you to subscribe to a monthly/yearly service plan, Circle of 6 has an unobtrusive screen that's simple to operate. Two taps will send out one of three Pre determined text messages to 6 contacts of your choice including a call for help getting home that automatically includes an address and map of your exact location, or a request for a phone call from you to break up a tense situation. The app also contains preprogrammed national hotline numbers and a local number you can customize for campus security police or 911. Circle of 6 is the winner of the Department of Health and Human Services / White House "Apps against Abuse" Challenge as well as for diabetics control measurement[2].

A wristband called Nymi, developed by Toronto-based company Bionym, confirms the wearer's identity through electrocardiogram (ECG) sensors that monitor the heartbeat, and then communicate that authentication to your devices ranging from IPads to cars[3]. Data logger built in with thousands of way point to record time stamp, speed, position, and event state with an press button only. Real time dynamic position report based on time interval or distance traveled.It is Zigbee based, it uses integrated ultra low power tilt sensor, also based on MQX real time operating system .New alert feature causes the transmitter to chirp loudly every time your child wanders beyond 20-30 foot range.

\section{SOFTWARE SPECIFICATION DETAILS}

For Front end Visual Basic . NET and for back end MSSQL is used.

Visual Basic .NET is a multipurpose computer programming language from Microsoft that is suitable for most development needs. The language is designed with Rapid Application Development in mind, providing several tools to shorten development time. The project will be implemented on visual Basic .NET , MS SQL due to features such as Buffer management , Logging and Transaction , Concurrency and locking, Replication services, Analysis services, Notification services, Integration services, Full text search service, Stored procedures.

\section{HARDWARE SPECIFICATION DETAILS}

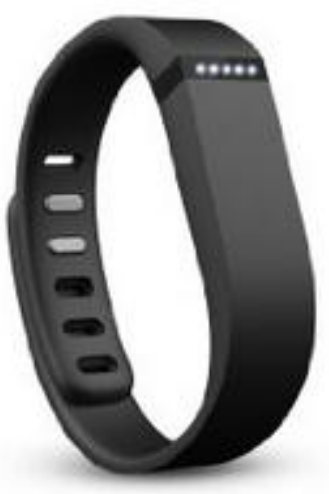

Fig 1: Hardware of wireless Bracelet 
The following are the hardware specifications used for this project :
i) $512 \mathrm{MB}$ ram
ii) $40 \mathrm{~GB}$ hard disk
iii) Pentium processor with LPT/COM/USB interface having $3 \mathrm{GHZ}$ and above processing speed
iv) Super hetero dyne transmitter and receiver[1]
v) Transistor driver

\section{METHOOLOGY}

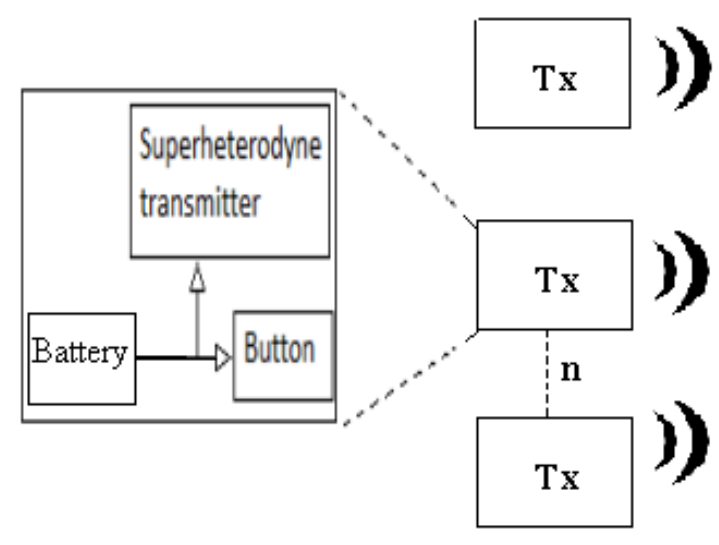

Fig 2 (a) : Transmitter part of Wireless Safety Bracelet

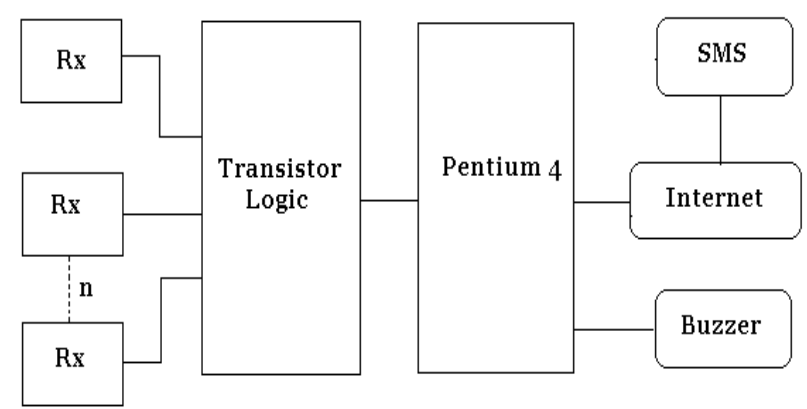

Fig 2(b) : Receiver part of Wireless Safety Bracelet

In our project, (Fig 2(a)) we are using bracelet which acts as a transmitter and it consist of transistor driver circuit, super heterodyne transmitter[2], which continuously provide radiations to receiver placed in one place of organization, as every person will have this bracelet so unique ID will be provided to each person.

Fig 2(b) indicate receiver part of wireless safety Bracelet.

In that , Buzzer is provided as if in case of emergency the person can press button and immediately the buzzer will produce a large alarm alert, and also immediately the activated contacts like police, family members the message will be sent and the women or any one can be helped as well as it will measure temperature and humidity within any organization and any condition comes then it will provide alarm [5].

The Pentium processors with MSSQL and .NET software's are used for this operation connected via. LPT interface cable , and at receiver, the display panel will provide information like time when the button was press, location. This all circuitry will be placed in certain one module that is bracelet.
On the transmit side the mixers convert the frequencies up instead of down.

For the receiver, the signal from the antenna is amplied in the radio frequency (RF) stage. The output of the RF stage is one input of a mixer. A Local Oscillator (LO) is the other input. The output of the mixer is at the Intermediate Frequency (IF). he concept here is that is much easier to build a high gain amplier string at a narrow frequency band than it is to build a wideband, high gain amplier. Also, the modulation bandwidth is typically very much smaller than the carrier frequency. A second mixer stage converts the signal to the baseband. The signal is then demodulated (demod). To put some numbers around it, let us consider a broadcast FM signal. The carrier frequency is in the range of $98 \mathrm{MHz}$ to $108 \mathrm{MHz}$. The IF frequency is almost always 10.7 MHz. The baseband is $0 \mathrm{~Hz}$ to $15 \mathrm{kHz}$. This is the sum of the right and left audio frequencies. There is also a modulation band centered at $38 \mathrm{kHz}$ that is the dierence of the left and right audio signals.

\section{OBJECTIVES}

The objective of this project are as follows :

I) To Provide Safety to individual within that range

ii) To check whether person is in organization or not.

iii) If any one is victimize then the information about its time, location will be available at receiver.

\section{SEQUENCE DIAGRAM}

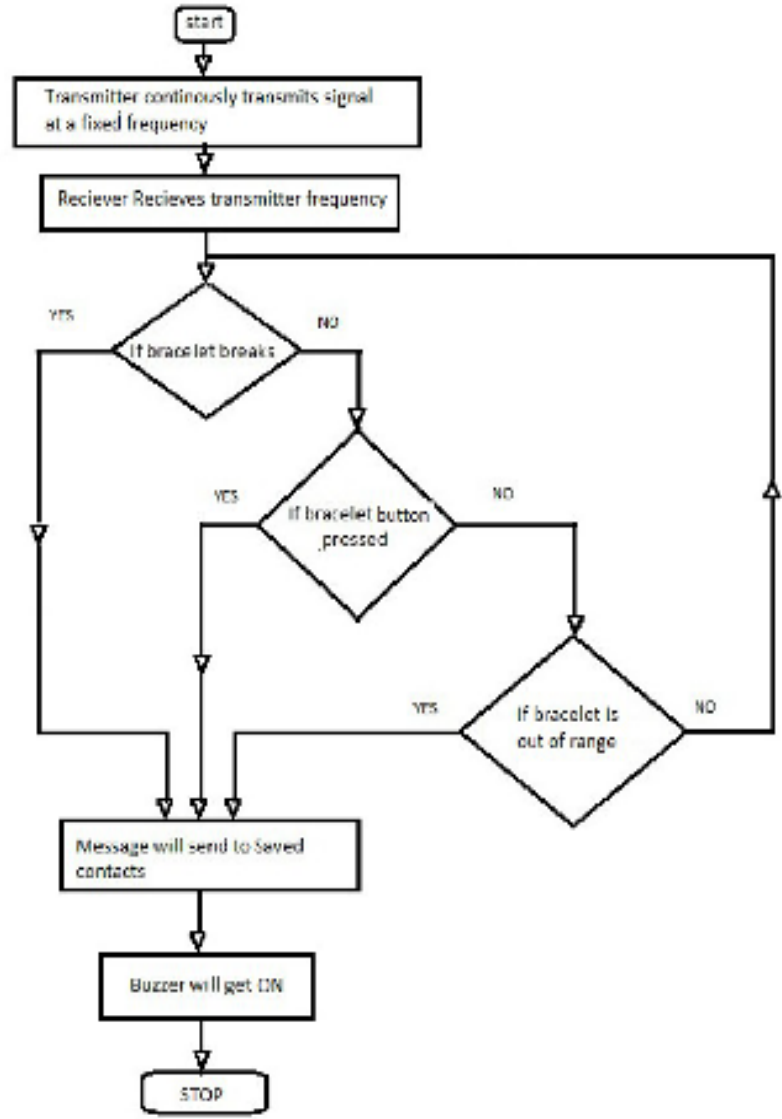

Fig 3: Sequence diagram of wireless safety Bracelet 
The figure above shows the sequence flow of wireless safety bracelet. The sequence is as follows :

\section{Start}

2. Bracelet will continuously transmits signal at a xed frequency.

3. Receiver will continuously receives transmitted frequency.

4. If bracelet breaks then go to step 7 else go to next step

5. If bracelet button get pressed then go to step 7 else go to next step

6. If bracelet is out of range then go to next step else go to step 4

7. Message will get send to saved contacts and buzzer will get 'ON'

\section{Stop.}

\section{APPLICATIONS \& USES}

The main application of this project is that it can be used for Wireless nurse call /duress alerting .

This project can also be implemented for following applications/uses :

I. Patient, resident identification

II. Mother/Baby matching

III. Senior living resident supervision

IV. Hands-Free access control

V. Entrance/Exit based alarming

VI. Efficiency and response reporting

\section{CONCLUSION}

In this Project we are going to implement such a product that will be work in an organization level only such as hospitals, collages etc. But still the question arises that what about the safety in remote area /? by taking this question into account we can make this device at such a extent so question about safety will not remain anymore by just adding such a module that will work in remote area.

\section{REFERENCES}

[1] www.news.cnet.com

[2] www.gizmag.com

[3] www.wikipedia.org

[4] www.sensorsmag.com

[5] Wikipedia, the free encyclopedia,"super heterodyne transmitter and receiver"

[6] J Med Internet Res. "Mobile applications for diabetics". 2014 Apr9;16(4):e104.doi:10.2196/jm7. SharonPros sharonpros ."Heartbeat detection using wrist band".May 22, 2014

[7] Conf Proc IEEE Eng Med Biol Soc. 2013 "A wearable healthcare system for cardiac signal monitoring using conductive textile electrodes." Hung SS, Chang CY, Hsu CJ,

[8] Chen SW. Epub 2012 Jun 29."Temperature and humidity sensors".

[9] Hung SS1, Chang CY, Hsu CJ, Chen SW Sensors (Basel). 2012 "Design of real timetemperature and humidity measurement system".

[10] Asis10 accolades "Tamper detection"

[11] Terry Chamber ,March 2011."LPT port" asp.net tutorials. "MSSQL".

[12] www.elpas.com "personal safety bracelet". 